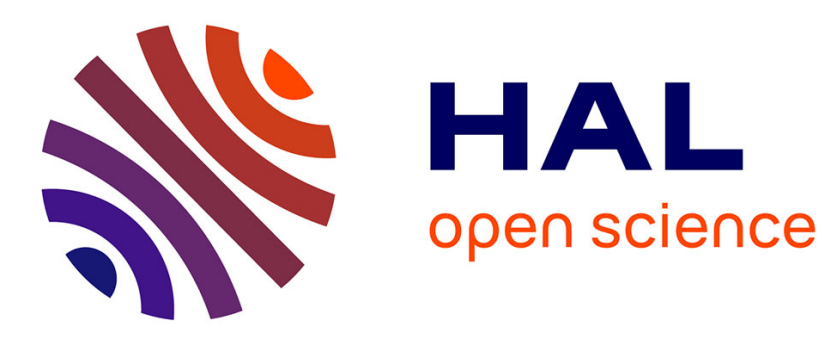

\title{
Multiuser underwater acoustic communication for an AUV fleet
}

\author{
Christophe Bernard, Pierre-Jean Bouvet
}

\section{To cite this version:}

Christophe Bernard, Pierre-Jean Bouvet. Multiuser underwater acoustic communication for an AUV fleet. OCEANS 2019 - Marseille, Jun 2019, Marseille, France. 10.1109/OCEANSE.2019.8867087. hal-03025517

\section{HAL Id: hal-03025517 https://hal.science/hal-03025517}

Submitted on 26 Nov 2020

HAL is a multi-disciplinary open access archive for the deposit and dissemination of scientific research documents, whether they are published or not. The documents may come from teaching and research institutions in France or abroad, or from public or private research centers.
L'archive ouverte pluridisciplinaire HAL, est destinée au dépôt et à la diffusion de documents scientifiques de niveau recherche, publiés ou non, émanant des établissements d'enseignement et de recherche français ou étrangers, des laboratoires publics ou privés. 


\title{
Multiuser underwater acoustic communication for an AUV fleet
}

\author{
Christophe BERNARD and Pierre-Jean BOUVET \\ SEACom lab \\ ISEN Brest YNCREA Ouest \\ Brest, France \\ Email: christophe.bernard@isen-ouest.yncrea.fr
}

\begin{abstract}
The objective of this paper is to investigate multiuser communication protocols in the framework of Underwater Acoustic (UWA) communication for Autonomous Underwater Vehicle (AUV)s fleet. By combining Hyperbolically Frequency Modulated (HFM) chirp and Pseudo-Noise (PN) spreading sequence we propose a very robust multi-user protocol that allows a very simple decoding based on matched filtering. The implementations of this protocol for the UWA scenario is described, and the performance results over a simulated shallow water UWA channel are compared against the conventional Code-Division Multiple Access (CDMA) and Time-Division Multiple Access (TDMA) protocols.
\end{abstract}

\section{INTRODUCTION AND MOTIVATIONS}

The UWA channel is one of the most challenging channel for data communications. Due to the low celerity of acoustic waves (with respect to electromagnetic ones), UWA channel is characterized by extensive multi-path effect and large Doppler spread. Moreover frequency dependent attenuation, temporal variation and background noise make UWA data rate very limited [1]. On the other hand, AUVs are used for several applications such as in military field with antisubmarine warfare, science field with wreck exploration, or in industrial field with offshore energy research. Nowadays the concept of a several AUVs working together within a fleet is an on-going research axis [2]. UWA communication with AUV fleet is used to control vehicles (downlink) or to gather data from vehicles (uplink). The quality and reliability of communication is essential, mainly in the shallow water areas for which the multi-paths effect is stronger leading to extensive intersymbol interference.

Traditional methods for multiuser communication in an underwater acoustic channel are TDMA and CDMA [3]. TDMA allows several users to share the same frequency channel by dividing the signal into different time slots. Each user uses alternatively its own time slot to transmit data without interfering with other users. However, as the number of users increases, the waiting time will be longer and the data rate will decrease. In CDMA protocol, the different users transmit information data simultaneously through a different spreading sequence for each user. The disadvantage of this method lies in the multiuser interference provided by the non-orthogonality of PN sequences when the user communication channel is selective in time or in frequency.
To cope with such interference terms, advanced equalization scheme can be invoked as multiuser detection [4] or MultiUser Multiple-Input Multiple-Output (MU-MIMO) technique combined with Passive Phase Conjugation (PPC) technique [5] but at the prize of higher decoding complexity and users number limitation. Recently, the authors of [6] propose an alternative of CDMA and TDMA by using chirp waveform for UWA multiuser communication. To reduce the multiuser interference, the Virtual Time Reverse Mirror (VTRM) technique is used with a Fractional Fourier Transform (FrFT) at the reception. However, this method requires an estimate of the different channels and is limited to 4 users.

In this paper, we introduce a new Multi-User Chirp Spread Spectrum (MU-CSS) method that does not require channel estimation and can be extended to more than 4 users. We consider an uplink scenario where a fleet of $N_{u}$ AUVs needs to transmit data to a receiver situated at the sea surface. The paper is organized as follows. System model and conventional multiuser protocols for UWA communications are introduced in section [II. The proposed MU-CSS multiuser scheme is presented in section IIII Simulation comparisons of the proposed scheme against conventional protocols are carried out in section $[\mathrm{IV}$ by using shallow water UWA channel simulator derived from [7], [8]. Finally, conclusions are drawn in section V.

\section{CONVENTIONAL MUlTIUSER ACCESS PROTOCOLS}

Multi-user UWA communication can be simply achieved by using TDMA protocol, but due to UWA channel characteristics, the time slot assigned to each user must have a substantial duration [3]. In the following, we will try to overcome limitations of TDMA protocol by considering simultaneous UWA transmission from each AUV.

\section{A. CDMA protocol}

A first classical approach consists of using spread spectrum modulation as CDMA [9] that is widely used as a multiple access method. CDMA assigns mutually orthogonal PN codes to different users. At the reception side, autocorrelation prop- 
erties and orthogonality between users of PN codes are used to dispread separately each user. For the $i$-th user, let:

$$
c_{i}(t)=\sum_{l=0}^{N_{S F}-1} c_{i, l} \phi\left(t-l T_{c}\right)
$$

with $\left[c_{i, 1}, c_{i, 2}, \ldots, c_{i, N_{S F}}\right]$ the PN length code of length $N_{S F}$ where $T_{c}$ is the chip duration, $S F$ the spreading factor and $\phi(t)$ is the pulse shaping filter. The baseband signal to be transmitted is then given by:

$$
s_{i}(t)=\sum_{k=1}^{N_{s}} d_{i, k} c_{i}\left(t-k T_{s}\right) \quad i \in\left[1, N_{u}\right]
$$

where $T_{s}=N_{S F} T_{c}$ is the symbol duration and $d_{i, k}$ are Differential Phase Shift Keying (DPSK) symbols carrying the useful information with $k \in\left[1, N_{s}\right]$. The use of differential coherent modulation is justified in our case because relative low data rate are considered in this paper. Moreover in UWA communication channel with large delay spreads and rapid time variations, differential modulation are demonstrated to provide interesting performance even to outperform coherent modulation under certain conditions [10].

The reception, the baseband received signal is expressed as:

$$
\begin{gathered}
r(t)=\sum_{i=1}^{N_{u}} h_{i}(t, \tau) * s_{i}(t)+n(t) \\
r(t)=\sum_{i=1}^{N_{u}} \int_{-\infty}^{+\infty} h_{i}(t, \tau) s_{i}(t-\tau) d \tau+n(t)
\end{gathered}
$$

with $h_{i}(t, \tau)$ the channel impulse response for the $i$-th user at time $t$ and $n(t)$ the additive Gaussian noise.

The transmitted symbols by the $i$-th user are found by matched filtering the received baseband signal with its PN code.

$$
\begin{aligned}
\hat{d}_{i}(t) & =c_{i}(t) \circ r(t) \\
& =\sum_{k=1}^{N_{s}} d_{i, k} h_{i}(t, \tau) * \rho_{i i}(t)+\zeta_{i}(t)+\tilde{n}(t)
\end{aligned}
$$

with

$$
\rho_{i, j}(t)=c_{i}^{*}(-t) * c_{j}(t)
$$

and

$$
\zeta_{i}(t)=\sum_{\substack{j=1 \\ j \neq i}}^{N_{u}} \sum_{k=1}^{N_{s}} d_{j, k} h_{j}(t, \tau) * \rho_{i j}(t)
$$

where $\zeta_{i}(t)$ represents the multi-user interference terms and $\tilde{n}(t)$ the residual noise.

To find the $k$-th symbol transmitted by the $i$-th user, we integrate on the symbol time:

$$
\hat{d}_{i, k}=\int_{-k \frac{T_{s}}{2}}^{k \frac{T_{s}}{2}} \hat{d}_{i}(t) d t
$$

\section{B. Direct Sequence Spread Spectrum (DSSS) TDMA}

As benchmark we consider a TDMA approach that uses a user time slot of $N_{s} T_{s}$ seconds followed by a guard interval of duration $T_{g}$ between each user slot. In order to deal with frequency selectivity of the UWA channel, a DSSS signalling is applied to each user of the TDMA protocol such as TDMA and CDMA approaches are equivalent in the single user scenario. The baseband received signal and the decoding process are given by particularizing (4) and (5) respectively with $N_{u}=1$.

\section{MU-CSS PROTOCOL}

\section{A. Concept}

To create the different spreading signals used by users, we will start from a HFM signal. The expression of the HFM signal is given by:

$$
x(t)= \begin{cases}\exp \left(-j 2 \pi k \log \left(1-\frac{t}{t_{0}}\right)\right) & \text { if } \frac{-T_{h}}{2} \leq t \leq \frac{T_{h}}{2} \\ 0 & \text { otherwise }\end{cases}
$$

with $t_{0}=\frac{T_{h}\left(f_{h}+f_{l}\right)}{2\left(f_{h}-f_{l}\right)}, k=\frac{T_{h} f_{l} f_{h}}{f_{h}-f_{l}}, f_{l} \leq f_{h}$ and $T_{h}$ the duration of the HFM signal.

Let $e_{i}(t)$ denote the signal corresponding to the $i$-th user with $i \in\left\{1,2, \ldots, N_{u}+1\right\}$ and $e_{1}(t)=x(t)$ (this signal will be excluded from the set later). The construction process is then defined for $i>1$ by:

$$
e_{i}(t)=c_{i}(t)+\alpha_{i} e_{i-1}(t)
$$

where

$$
\alpha_{i}=\frac{<c_{i}(t), e_{i-1}(t)>}{\left\|e_{i-1}(t)\right\|_{2}}=\frac{\int_{\frac{-T_{h}}{2}}^{\frac{T_{h}}{2}} c_{i}(t) e_{i-1}^{*}(t) d t}{\left\|e_{i-1}(t)\right\|_{2}}
$$

with $c_{i}(t)$ given by equation (1).

\section{B. User decoding}

The baseband signal to be transmitted is given by:

$$
s_{i}(t)=\sum_{k=1}^{N_{s}} d_{i, k} e_{i}\left(t-k T_{h}\right) \quad i \in\left[1, N_{u}\right]
$$

with $d_{i, k}$ are the DPSK symbols carrying the useful information with $k \in\left[1, N_{s}\right]$. At the reception, the baseband received signal is the same as equation (3) and the transmitted symbols by the $i$-th user are found by matched filtering the received baseband signal with its corresponding chirp.

$$
\begin{aligned}
\hat{d}_{i}(t) & =e_{i}(t) \circ r(t) \\
& =\sum_{k=1}^{N_{s}} d_{i, k} h_{i}(t, \tau) * \rho_{i i}(t)+\zeta_{i}(t)+\tilde{n}(t)
\end{aligned}
$$

with

$$
\rho_{i, j}(t)=e_{i}^{*}(-t) * e_{j}(t)
$$


and

$$
\zeta_{i}(t)=\sum_{\substack{j=1 \\ j \neq i}}^{N_{u}} \sum_{k=1}^{N_{s}} d_{j, k} h_{j}(t, \tau) * \rho_{i j}(t)
$$

where $\zeta_{i}(t)$ represents the multi-user interference terms and $\tilde{n}(t)$ the residual noise.

To find the $k$-th symbol transmitted by the $i$-th user, we integrate on the chirp time:

$$
\hat{d}_{i, k}=\int_{-k \frac{T_{h}}{2}}^{k \frac{T_{h}}{2}} \hat{d}_{i}(t) d t
$$

\section{Simulation REsults}

\section{A. Underwater acoustic channel simulator}

For the simulation comparisons, we consider the UWA channel simulator provided by [7] based on a stochastic model. The time varying transfer function is given by:

$$
H(f, t)=\bar{H}_{0}(f) \sum_{p} \gamma_{p}(f, t) h_{p} e^{-j 2 \pi f \tau_{p}}
$$

with $\bar{H}_{0}(f)$ the transfer function of direct path, $h_{p}$ the relative path gain, $\gamma_{p}(f, t)$ represents the scattering coefficient modeled by a complex-valued Gaussian processes whose statistics reflects the time coherence of the channel and $\tau_{p}$ the path delays. The time variation of $\gamma_{p}(f, t)$ leads to Doppler spread. For simplicity, we will take $\gamma_{p}(f, t) \sim \mathcal{N}\left(0,10^{-10}\right)$ [8]. In this study we assume that the AUVs have no intentional motion.

\section{B. System parameters}

The chosen model represents a short range UWA transmission with a water depth $10 \mathrm{~m}$ at a center frequency of $23 \mathrm{kHz}$ over a $4 \mathrm{kHz}$ bandwidth. Each AUV are supposed at a same depth of $1 \mathrm{~m}$. The range between each AUV and the receiver is randomly selected in the interval $[700,710] \mathrm{m}$ modeling a fleet situating in a circular ring. In a first time, we will analyze the results for a static channel and in a second time we will use a time varying channel.

Channel parameters are summarized in Table I whereas transmission system parameters are provided in table II

\begin{tabular}{|l|c|}
\hline Parameters & Value \\
\hline Center frequency & $23 \mathrm{kHz}$ \\
\hline Sample frequency & $100 \mathrm{kHz}$ \\
\hline Signal bandwidth & $4 \mathrm{kHz}$ \\
\hline Transmission range & {$[700,710] \mathrm{m}$} \\
\hline Water depth & $10 \mathrm{~m}$ \\
\hline RMS channel delay spread 11] & $16 \mathrm{~ms}$ \\
\hline Signal to noise ratio & $10 \mathrm{~dB}$ \\
\hline Doppler spread & $N\left(0,10^{-10}\right)$ \\
\hline
\end{tabular}

TABLE I

CHANNEL PARAMETERS.

To compute the Frame Error Rate (FER), we consider a frame as erroneous when the Bit Error Rate (BER) after

\begin{tabular}{|l|c|r|}
\hline Symbol & Signification & Value \\
\hline$M$ & Modulation & 1 (DBPSK) \\
\hline$N_{s}$ & Number of symbols & 200 \\
\hline$N_{f}$ & Number of frames & $10^{3}$ \\
\hline $\mathcal{C}$ & Forward Error Correction (FEC) code type & Convolutive code \\
\hline$g_{\mathcal{C}}$ & FEC code generator & $(156,123)_{o}$ \\
\hline$R_{\mathcal{C}}$ & FEC code rate & $15 \mathrm{~ms}$ \\
\hline$T_{g}$ & Guard interval time & $7.75 \mathrm{~ms}$ \\
\hline$T_{h}$ & Duration of the chirp signal & $? ? ? \mathrm{~ms}$ \\
\hline$T_{c}$ & chip duration & 31 \\
\hline$N_{S F}$ & PN length code & $? ? ? ?$ \\
\hline$\alpha$ & pulse shaping filter roll-off factor & $7.75 \mathrm{~ms}$ \\
\hline$T_{s}$ & Symbol time & \\
\hline
\end{tabular}

TABLE II

SYSTEM PARAMETERS

channel decoding is greater than $10^{-3}$. The effective data rates for each protocols are given below:

$$
\begin{gathered}
D_{e}^{\mathrm{CDMA}}=\frac{R_{\mathcal{C}} \log _{2} M}{N_{S F} \cdot T_{c}} \cdot(1-\mathrm{FER}) \quad[\mathrm{bps}] \\
D_{e}^{\mathrm{TDMA}}=\frac{N_{s} R_{\mathcal{C}} \log _{2} M}{N_{s} N_{u} N_{S F} T_{c}+\left(N_{u}-1\right) T_{g}} \cdot(1-\mathrm{FER}) \\
D_{e}^{\mathrm{MU}-\mathrm{CSS}}=\frac{R_{\mathcal{C}} \log _{2} M}{T_{h}} \cdot(1-\mathrm{FER}) \quad[\mathrm{bps}]
\end{gathered}
$$

where $M$ is the size of the DPSK constellation and $R_{\mathcal{C}}$ is channel coding rate.

\section{Static channel}

In a first step we consider a static channel Simulation comparisons between the protocols over the modeled shallow water acoustic static channel are given by figure 1 and figure 2. As expected the TDMA protocol provides perfect FER

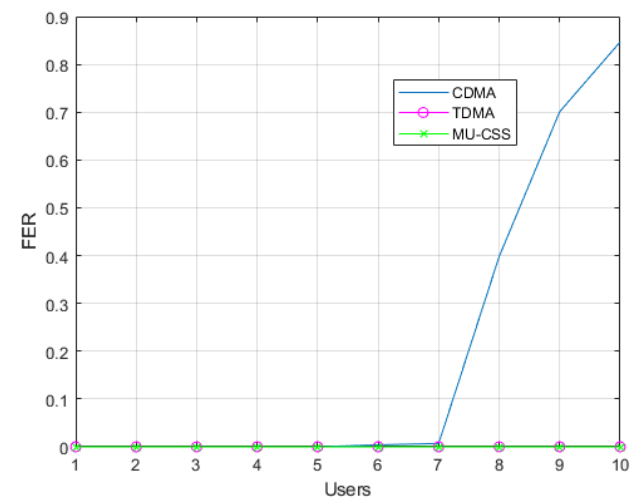

Fig. 1. Average FER performance versus number of users for the static channel.

whatever the number of users whereas robustness of CDMA decreases as the number of users increases. By analyzing the effective data rate, CDMA is demonstrated to reach higher data rate than classical TDMA approach. The MU-CSS protocol have a perfect FER as the TDMA and they have the best effective data rates. 


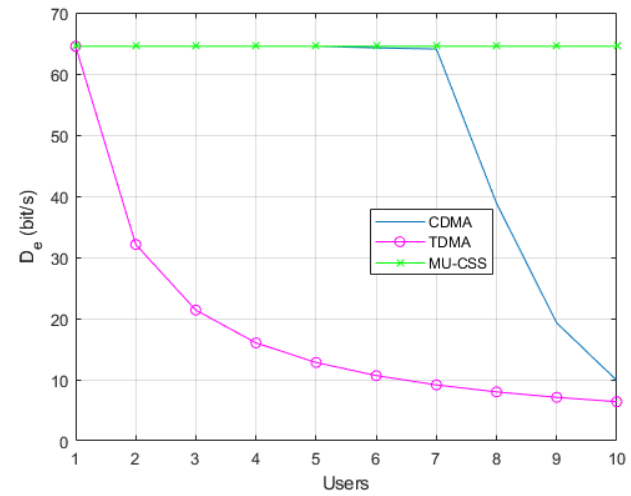

Fig. 2. Average effective data rate per user versus number of users for the static channel.

\section{Time varying channel}

An a second step, Doppler spread is added in shallow water UWA channel model leading to figure 3 and figure 4. Over time varying channel, the effective data rate of the

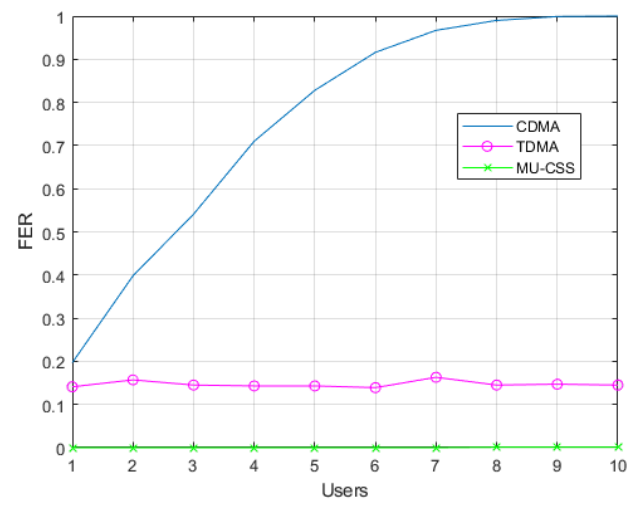

Fig. 3. Average FER performance versus number of users for the time varying channel.

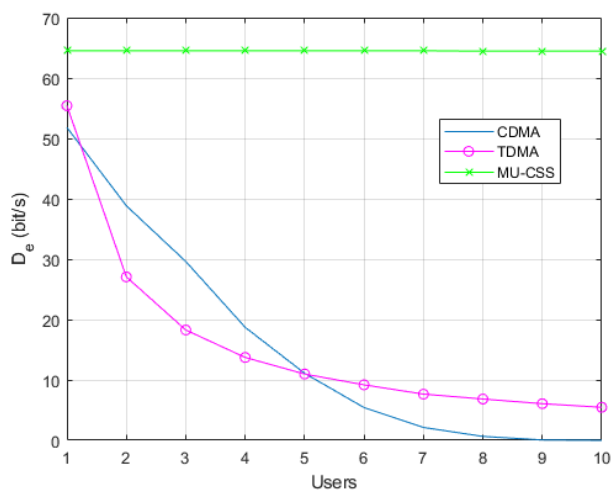

Fig. 4. Average effective data rate per user versus number of users for the time varying channel.

conventional access protocols is decreased. We can see TDMA performance are getting higher than CDMA beyond 5 users. In fact, TDMA protocol is not affected by multiuser interference but only UWA channel time and frequency selectivity while CDMA suffers from multiuser interference in addition to UWA channel selectivity. The MU-CSS protocol have the best effective data rates compared to other protocols.

\section{CONCLUSION}

In this paper, we have proposed a new multiuser protocol denoted MU-CSS in the context of UWA communication within an AUVs fleet. Simulation comparisons against traditional TDMA and CDMA over static and time-varying shalow water UWA models demonstrate a superior effective data rate for the proposed MU-CSS scheme even if the number of users is large.

In a future work, we will analysis the behavior of the multiuser protocols in the case of large and different Doppler shifts induced by the motion of each AUV as well as sea experiments.

\section{REFERENCES}

[1] M. Stojanovic and P.-P. J. Beaujean, "Acoustic Communication," in Springer Handbook of Ocean Engineering, M. R. Dhanak and N. I. Xiros, Eds. Springer International Publishing, 2016, pp. 359-386.

[2] B. T. Champion and M. A. Joordens, "Underwater swarm robotics review," 2015 10th System of Systems Engineering Conference (SoSE), pp. 111-116, May 2015.

[3] R. Otnes, A. Asterjadhi, P. Casari, M. Goetz, T. Husy, I. Nissen, K. Rimstad, P. v. Walree, and M. Zorzi, Underwater Acoustic Networking Techniques, ser. SpringerBriefs in Electrical and Computer Engineering. Berlin Heidelberg: Springer-Verlag, 2012. [Online]. Available: //www.springer.com/la/book/9783642252235

[4] M. Stojanovic and L. Freitag, "Multichannel Detection for Wideband Underwater Acoustic CDMA Communications," IEEE Journal of Oceanic Engineering, vol. 31, no. 3, pp. 685-695, Jul. 2006.

[5] T. C. Yang, "Spatially Multiplexed CDMA Multiuser Underwater Acoustic Communications," IEEE Journal of Oceanic Engineering, vol. 41, no. 1, pp. 217-231, Jan. 2016.

[6] Q. W. F. Yuan and E. Cheng, International Journal of Naval Architecture and Ocean Engineering.

[7] P. Qarabaqi and M. Stojanovic, "Statistical Characterization and Computationally Efficient Modeling of a Class of Underwater Acoustic Communication Channels," IEEE Journal of Oceanic Engineering, vol. 38, no. 4, pp. 701-717, Oct. 2013.

[8] Y. M. Aval, S. K. Wilson, and M. Stojanovic, "On the Achievable Rate of a Class of Acoustic Channels and Practical Power Allocation Strategies for OFDM Systems," IEEE Journal of Oceanic Engineering, vol. 40, no. 4, pp. 785-795, Oct. 2015.

[9] R. Z. R.L. Peterson and D. Borth, Introduction to spread-spectrum communications. Prentice Hall, 1995.

[10] Y. M. Aval, S. K. Wilson, and M. Stojanovic, "On the Average Achievable Rate of QPSK and DQPSK OFDM Over Rapidly Fading Channels," IEEE Access, vol. 6, pp. 23 659-23 667, 2018.

[11] J. G. Proakis and M. Salehi, Digital Communications 5ed, 5th ed. Boston, Mass.: McGraw-Hill, 2008. 